\section{Fehler, zufälliger}

R.-D. Hilgers ${ }^{1}$, N. Heussen ${ }^{1}$ und S. Stanzel ${ }^{2}$

${ }^{1}$ Institut für Medizinische Statistik, Universitätsklinikum der RWTH Aachen, Aachen, Deutschland

${ }^{2}$ DKFZ Heidelberg, Heidelberg, Deutschland

Synonym(e) Fehler; Zufällige Messabweichung

Englischer Begriff random error

Definition Unter einem zufälligen Fehler ( $\vee$ Fehler, zufälliger) versteht man die zufällige $\triangleright$ Variabilität der Messergebnisse bei der mehrmaligen Durchführung von Messwiederholungen.
Beschreibung Die Ursachen für das Auftreten zufälliger Fehler sind im Einzelnen nicht bekannt und daher nicht analysierbar. Das Wesen zufälliger Fehler besteht darin, dass Abweichungen von einem Sollwert sich im Mittel ausgleichen ( $\triangleright$ Mittelwert, arithmetischer) und nicht $\mathrm{zu}$ „starke“ Variabilität auftritt. Mithilfe der $>$ Präzisionskontrolle können zufällige und systematische Fehler ( $\vee$ Messabweichung, systematische) voneinander abgegrenzt werden.

\section{Literatur}

Qualitätskontrolle im Medizinischen Laboratorium von A bis Z - Ein Leitfaden in Schlagworten, 2., überarb. Aufl. Behring Diagnostika https://doi.org/10.1515/labm.1995.19.1-12.218 